\title{
Adaptive Gaussian Distribution Threshold Spatial Frequency Denoising Image
}

\author{
${ }^{1}$ BENZIANE SARAH, ${ }^{2}$ BENYAMINA ABOU EL HASSEN \\ ${ }^{1}$ Université des sciences et de la technologie d'Oran, Département d'informatique, Oran, ALGERIA \\ ${ }^{2}$ Université Oran -1- Ahmed Ben, Bella, Département d'informatique, Oran, ALGERIA \\ LAPECI Laboratory
}

\begin{abstract}
The details of an image with noise can be restored by removing the noise with an appropriate image denoising method. In this work is proposed and tested, an image denoising methods based on the use of an improved generalized adaptive Gaussian distribution threshold in the wavelet domain. Different wavelet transform methods are used in conjunction with an AGGD threshold to experiment with the proposed approach in order to obtain better results for the image denoising process and, consequently, to select the most suitable filter. The wavelet transform working on the frequencies of separate subbands of an image is a powerful method for image analysis. According to this experimental work, the proposed method show better results. The MSE and PSNR values get are used to measure the enhancement of denoised images.
\end{abstract}

Keywords- Denoising, satellite image, spatial frequency, gauss, wavelets, Gaussian distribution, grayscale, RGB, decision trees, RF Random Forest.

Received: April 26, 2021. Revised: November 21, 2021. Accepted: December 14, 2021. Published: December 29, 2021.

\section{Introduction}

Nowadays, most remote sensing data is recorded in digital format and almost all image interpretation and analysis requires some digital image processing. The latter can use various processes including formatting and data correction, as well as digital enhancement to facilitate visual interpretation or even automatic classification of targets and structures entirely by computer.

When we look at satellite images as an example of the Algerian Alsat-1 satellite, we intuitively see that they suffer from two main problems: low contrast and the presence of noise of which we have no prior knowledge. The denoising of these images is examined as a real case of blind restoration where one tries to estimate the noise before the denoising process. Others estimate, it is based on the principle of edge detection in the spatial domain, and is used to calculate the threshold value required to estimate the informative wavelet coefficients. To do this, we have divided our work into three sections:

The first section provides a general and inevitably superficial overview on the formulation of the image denoising problem. Then, is presented several classical and modern image denoising techniques.

The second section introduces an overview on the ondelette transform, in particular the multi-resolution analysis. We implemented a technique based on spatial frequency denoising with improved generalized adaptive Gaussian distribution threshold in the wavelet domain, This approach is applied to Landsat images in order to be able to evaluate their efficiency by quantitative measures such as the mean squared error or signal-to-noise ratio.

In the third section, we present a technique based on decision trees in this case Random forest RF. A method of denoising still images by supervised machine learning for the estimation of informative wavelet coefficients. Finally, we will conclude this work by highlighting its important points, and emphasizing its limits, and by evoking some research perspectives as a guide to improvement.

\section{Section I: State of the Art of Previous Work}

Since the early 1990s, image denoising has grown significantly. Even today, new methods are born and developed around many different heuristics and theories.

\subsection{Noise}

It is a random variation of the brightness or color information in the images and an unwanted by-product of the image that obscures the desired information.

Generally, noise is introduced into the image during the steps of transmission, acquisition, coding or processing of the image.

- Additive noise - where image noise is added to the original image to produce a corrupted noisy image. $C(x, y)=O(x, y)+N(x, y)$.

- Multiplicative noise - where the image noise is multiplied by the original image to produce a corrupted noisy image. $C(x, y)=O(x, y) * N(x, y)$.

Hence: $\mathrm{C}(\mathrm{x}, \mathrm{y})=$ noisy image; $\mathrm{O}(\mathrm{x}, \mathrm{y})=$ Original image; $\mathrm{N}$ $(\mathrm{x}, \mathrm{y})=$ image noise; [1]

\subsection{Image Denoising Methods}

There are two fundamental bases or methods of image denoising: [2]

(1) Spatial domain methods. 
(2) Transformation domain methods.

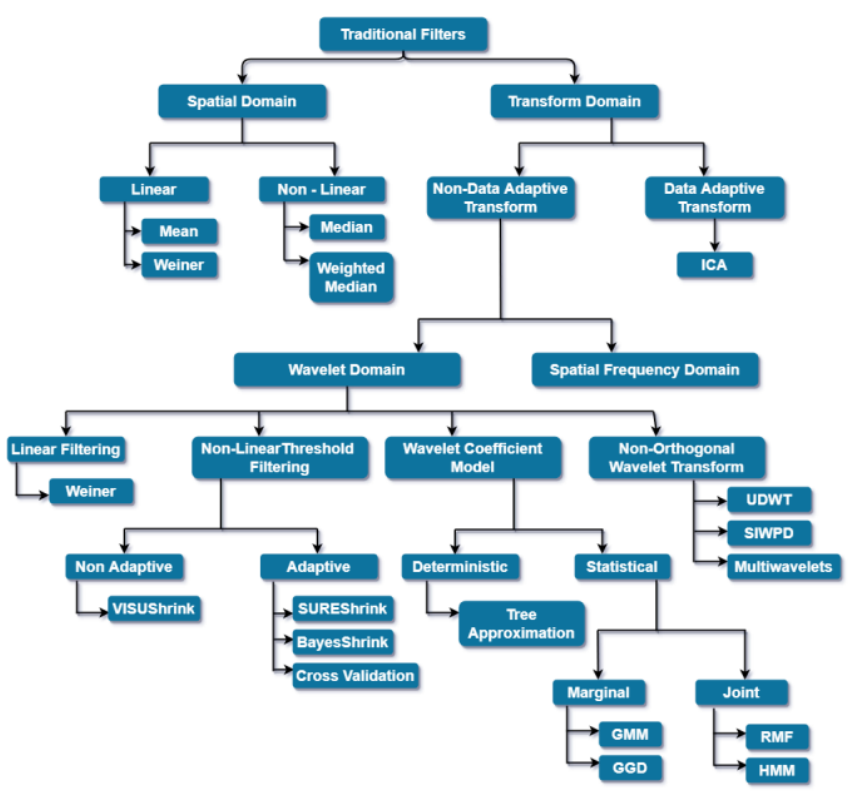

Figure 01: Image denoising techniques

Wavelet transform has been used by many researchers to denoise corrupted photos as well as compress them.

Some have changed the wavelet bases, changed the thresholding procedure, merged additional filtering approaches, and compared the performance of their suggested methods to that of other well-known methods. [9] have compared haar and DB3 wavelets for the removal of speckle noise from ultrasound, MRI, X-ray, and CT scan images; [10] has compared Haar, Daubachies, symllet, and coiflet wavelets for eliminating additive white Gaussian noise with various thresholding strategies;[11] for removing additive white Gaussian noise, have estimated the threshold and neighbouring window size for subband using its length for visuShrink, Modineighshrink, and Neighshrink in wavelet transform; [12] Different wavelet transform filtering strategies for denoising Gaussian, Salt and Pepper, and speckle noise from Lena picture were examined. [13] For removing Gaussian, Poisson, Salt and Pepper, and speckle noise from an image, we changed the fundamental thresholding method and used circular kernel, Mean max approximation, and Nearest Neighbor techniques; [14] In order to denoise speckle noise in the Lena image, we employed Weighted variance to compute the threshold in the wavelet domain. [15] Sub band decomposition of logarithmically transformed images represented by alpha stable distributions was used to calculate the threshold for reducing speckle noise in ultrasound images, which was subsequently used by the Bayesian estimator. [16] have developed an adaptive threshold estimation approach for removing Gaussian noise from Lena, Goldhill, and Barbara pictures based on the Generalized Guassian distribution (GGD) modeling of subband coefficients (NormalShrink); [17] For removing Gaussian noise from the Lena image, we used a variety of wavelet bases and performed Wavelet transformations from the second to fourth level decomposition, as well as thresholding techniques such as Visushrink, Neighshrink, and Modineighshrink. [18] For removing Gaussian from Pepper, Barbara, Crowd, Goldhill, Boat, Bridge, and $\mathrm{Al}$ pictures, we used Stein's unbiased risk estimate and interscale orthonormal wavelet thresholding technique; [19]For eliminating AWGN from 3D pictures, they employed the Laplacian pyramid and the windowed Fourier transform; [20] Multi-wavelet transformation was used to remove AWGN from mammographic images; [21] have utilized the Contourlet transform to remove AWGN from brain and spine MRI images;[22] developed a new approach for getting image denoising thresholds using wavelet soft-thresholding that is data-dependent (Bayes Shrink), as well as attempting to compress the image using MDLQ during denoising.[23] Salesnick suggested an expanded least square technique to $2 \mathrm{D}$ pictures for noise removal that used wavelet coefficients instead of second order filter coefficients.

\section{Section Ii: Multi-scale Noise Reduction in the Wavellet Field}

Within the framework of the image denoising, we are interested in the denoising of remote sensing images with TO, while noting that the studied methods can be generalized in restoration schemes of different types of images. By way of example, we will apply the studied methods to some images of the Landsat satellite.

The Wavelet Transform (TO) is a mathematical tool which decomposes a signal into frequencies while maintaining a temporal localization. It offers great design flexibility and efficient signal representation, finely tuned to its intrinsic properties. By combining these observations with simple processing techniques in the transformation field, multiscale analysis can accomplish remarkable performance and efficiency at many image processing problems, in addition to its rapid implementation due to the use of filter banks which allow a real processing capacity in time.

The representation of a continuous signal $x(t)$ by $a$ wavelet base is given by: [2]

$$
x(t)=\sum C(s, \tau) \varphi_{s \tau}(t)
$$

where $\mathrm{C}(\mathrm{s}, \tau)$ is the weight of the contribution of the wavelet $\varphi \_s \tau$ linked to the mother wavelet by:

$$
\varphi_{s \tau}(t)=\frac{1}{\sqrt{s}} \varphi\left(\frac{t-\tau}{s}\right)
$$

where: $\tau$ : translation factor; $s$ : scale factor; $\varphi$ : mother wavelet; $\frac{1}{\sqrt{s}}$ : energy normalization factor so that the transformed signal has the same energy at all scales.

\subsection{7 he Continuous Wavelet Transform}

The Continuous Wavelet Transform (TOC) uses translations and dilations of the mother wavelet function throughout the time interval in a continuous manner [3]. It is defined by:

$$
W[x(t)]=C(s, \tau)=\frac{1}{\sqrt{s}} \int_{-\infty}^{+\infty} x(t) \varphi\left(\frac{t-\tau}{s}\right) d t
$$

Thus, the weighting $\mathrm{C}(\mathrm{s}, \tau)$, also called wavelet coefficients and defined by (1), is none other than the Wavelet transform of the function $\mathrm{x}(\mathrm{t})$. This transformation is in theory infinitely redundant since the wavelet is continuously translated.

\subsection{7 he Discrete Wavelet Transform}

It is pretty much obvious that neither TF, STFT, nor TOC can be manually calculated using analytical equations, integral calculus, etc. It is therefore up to computers to 
calculate these transforms. In the case of TOC, one can use the variation of the scale to reduce the sampling frequency. [4] Discrete Waveform Transform (TOD) is produced to overcome the redundancy problem of TOC. This redundancy mobilizes a large amount of computational resources. TOD, on the contrary, provides sufficient information, both for the analysis and for the reconstruction of the original signal, in a significantly reduced computation time. [5]

\subsection{Transformed Into Two-dimensional Wavelets}

The two-dimensional extension of the TOD (TOD-2D) is essential for the transformation of two-dimensional (2D) signals, such as a digital image. The simple approach for implementing TOD-2D is to perform one-dimensional TOD (TOD-1D) row by row to produce an intermediate result and then perform the same column TOD-1D on that intermediate result to get the final result

The TOD-2D can be interpreted as the decomposition on a set of spatially oriented frequency channels.

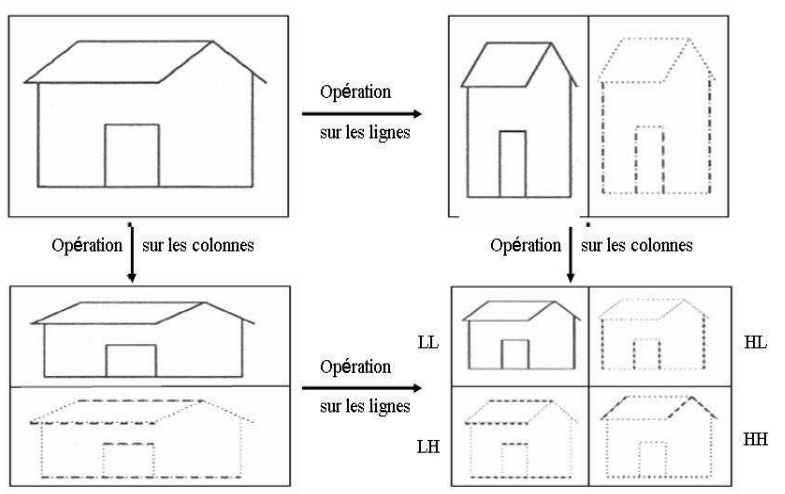

Figure 02: Model of the 2D multi-resolution analysis [26].

When we iterate on the low frequency signals, we obtain several levels of wavelet decomposition. After the first level of decomposition in figure 03 .

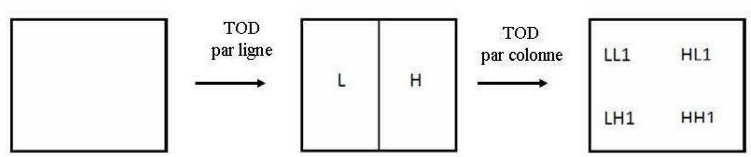

(a) Premier niveau de décomposition

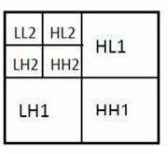

(b) Deuxième niveau

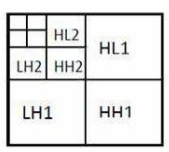

(c) Troisième niveau
Figure 03: Representation of the TOD of an image[26].

The filtering procedures for $2 \mathrm{D}$ wavelet decomposition and reconstruction are shown in Figures 04 and 05 respectively.

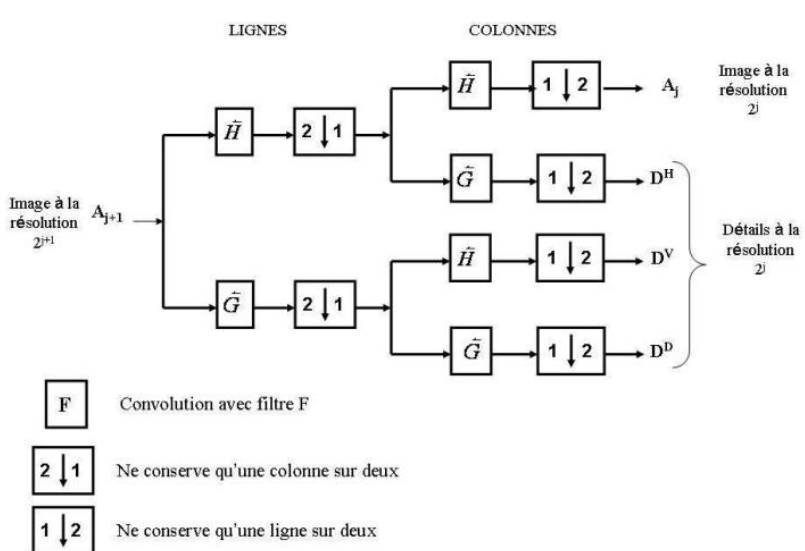

Figure 04: A level of multi-resolution representation of an image[26].

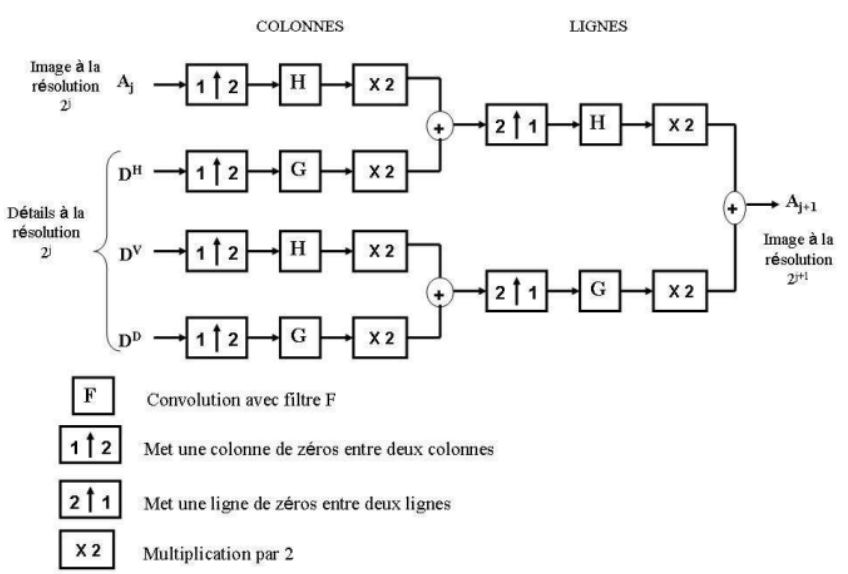

Figure 05: A level of reconstruction[26].

\subsection{7 he Used Method}

1. Read the test image (original).

2. Resize the test image and convert it to a grayscale image.

3. Noise of the desired level is mixed with the test images. The pixel value modified by additive Gaussian noise can be represented as follows:

4. $J(k, l)=x(k, l)+n$

5. Where is the noise. $\mathrm{n}=\mathrm{N}(0, \mathrm{v})$ being distributed normally with the variance $\mathrm{v}$.

6. Have the noisy image undergo a discrete wavelet transformation, DWT.

7. Once the noisy image has been decomposed into approximation and detail coefficients using the Wavelet Transform. It is subject to our proposed modified thresholding rules having varying threshold values. The BayesShrink Modified Threshold is an adaptive threshold that is modified and extended up to three different levels. By setting the derivative of the risk to zero with respect to the optimal threshold we find:

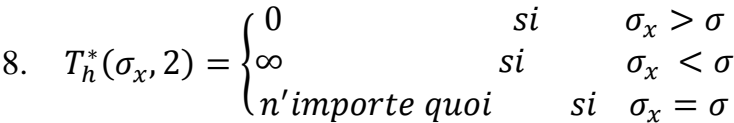

With the associated risk 
$r_{h}\left(T_{h}^{*}\right)=\left\{\begin{array}{lll}\sigma^{2} & \text { if } & \sigma_{x}>\sigma \\ \sigma_{x}^{2} & \text { if } & \sigma_{x} \leq \sigma\end{array}\right.$

9. After the decomposition, the coefficients are thresholded using the threshold values mentioned above with each of the thresholding techniques, the denoised image is reconstructed using the IDWT Inverse Wavelet Transform. [6]

10. Calculate the various standard performance parameters Like MSE. PSNR, which are calculated for all standard images with their noisy and denoised images.

\section{Section Iii: Noise Reduction by Random Forest in the Wavellet Area}

The "random forests" algorithm was proposed by Leo Breiman and Adèle Cutler in 2001 [7]. In its most classic formula, is a supervised machine learning algorithm that is widely used in classification and regression problems. It performs parallel learning on multiple decision trees randomly constructed and trained on subsets of different samples and takes their majority vote for ranking and average when the data is quantitative or used for voting for qualitative data, in the case of classification trees. [25]

The Random Forest algorithm is known to be one of the most efficient "out-of-the-box" classifiers (that is, requiring little data preprocessing), one of its most important features. is that it can handle the dataset containing continuous variables as in the case of regression and categorical variables as in the case of classification. It performs better for classification problems and has been used in many applications, including consumer applications, such as image denoising. [26].

Bagging, also known as bootstrap aggregation [8], is the ensemble technique used by random forest. Bagging chooses a random sample from the data set. Therefore, each model is generated from the samples (Bootstrap Samples) provided by the original data with a replacement known as inline sampling. This step of sampling rows with replacement is called a bootstrap. Now each model is trained independently, which generates results. The end result is based on majority voting after combining the results of all models. This step of combining all of the results and generating an output based on a majority vote is known as aggregation.

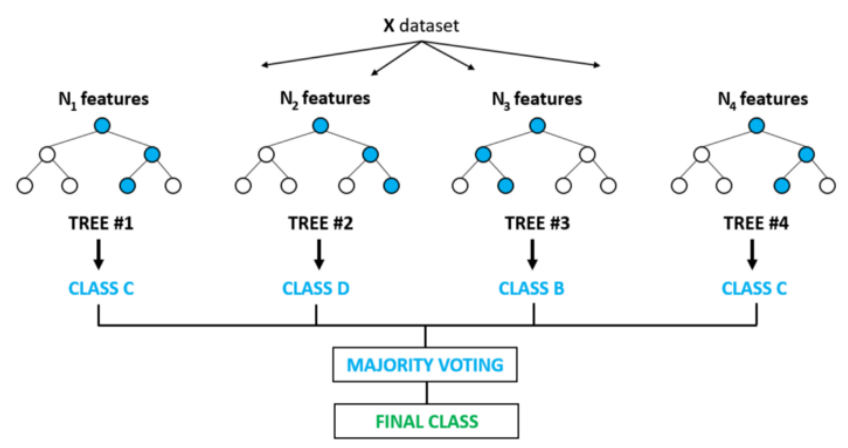

Figure 05: A representation of the random forest

\section{Result and Discussions}

\subsection{Summary of Results}

Table 01: shows a summary of the results of PSNR simulations obtained through this work. The proposed method is compared with the method proposed in section 2 and the VisuShrink method.

Table 1: Results parameters

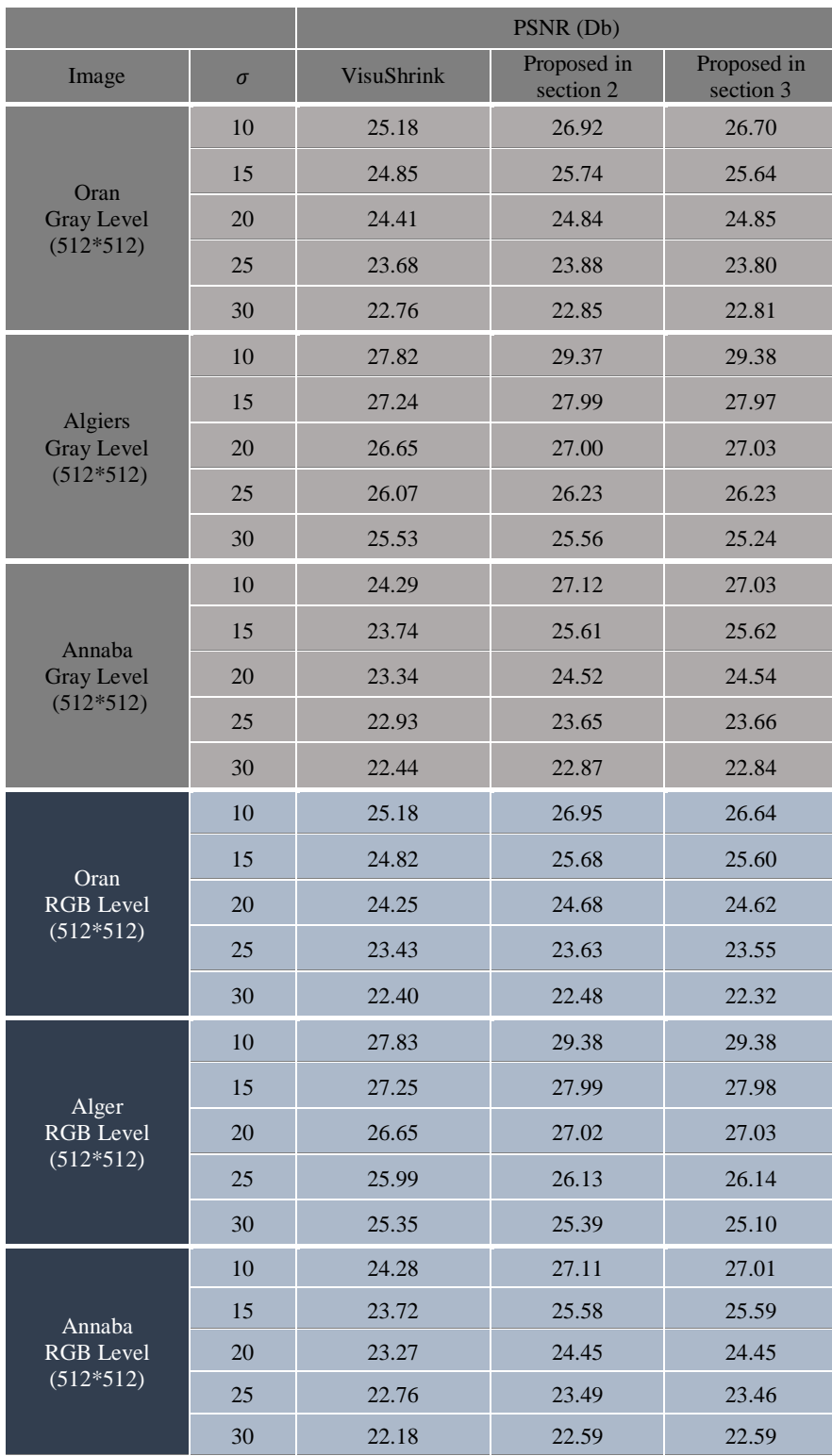

\subsection{Samples of the Denoised Images}
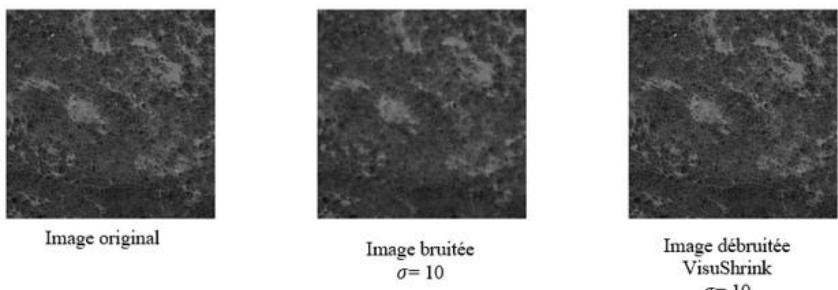

Image débruitée $\sigma=10$

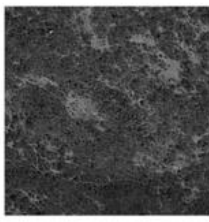
Image débruitée
AGGD Bayes $\begin{aligned} \sigma & =10 \\ \text { PSNR } & =26.92\end{aligned}$ $\sigma=10$ $\begin{aligned} \sigma & =10 \\ \operatorname{PSNR} & =25.18\end{aligned}$

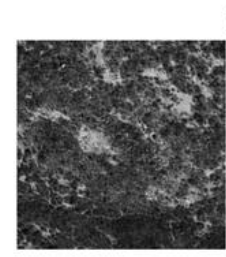

$$
\begin{gathered}
\text { Image débruitée } \\
\text { RF } \\
\sigma=10 \\
\text { PSNR }=26.70
\end{gathered}
$$

Figure 06: Estimation of the noise of the image of Oran, Alsat-1 satellite in gray level with $\sigma=10$ 


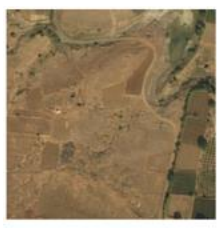

Image Original

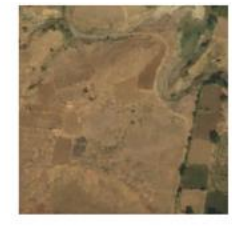

Image bruitée

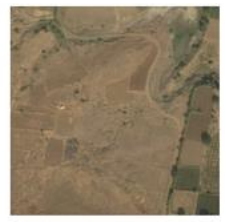

Image débruitée isuShrimk
$\sigma=25$ $\begin{aligned} \sigma & =25 \\ \operatorname{PSNR} & =25.99\end{aligned}$

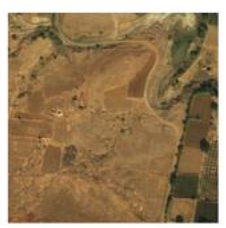

Image débruitée $\sigma=25$
$\mathrm{PSNR}=26.13$ Image debruitee
AGGD BayesShrink
$\sigma=25$
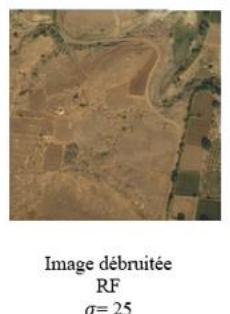

$\sigma=25$
PSNR $=26.14$
Figure 07: Estimation of the image noise of Algiers, Alsat-1 satellite in RGB level with $\sigma=25$

\subsection{Discussion of the Results}

In order to carry out a complete comparative study, we used the denoising methods given by the thresholding. Examination of the tables presented in this section shows the efficiency of the method proposed in the second section compared to the VisuShrink method for all image sizes and also when the noise variance is high.

Table 01 and Figures [06 - 07] show that the AGGD method gives better objective and subjective performance than the other methods studied; this can be interpreted by the use of adaptive methods when calculating the Visushrink method.

Columns 3 of Table 1 represent the classical technique of wavelet thresholding (VisuShrink). Thresholding consists of canceling all the wavelet coefficients below a threshold. As we mentioned before. This process exists in two forms: hard thresholding consists in leaving the wavelet coefficients below the threshold unchanged; on the other hand soft thresholding modifies them by reducing their amplitude. Images obtained after reconstruction generally contain inhomogeneous information and high levels of noise. The wavelet reconstruction generates artefacts on such images (see figure [06 - 07]).

It appears too that the results obtained by the "BayesShrink" method are better than those obtained by conventional thresholding methods in terms of PSNR and visual quality.

These performances can be interpreted by the fact that the "BayesShrink" method uses an adapted threshold for each level of decomposition unlike the conventional thresholding which uses a common (universal) threshold for all the levels.

With regard to the methods, BayseShrink and the Random Forest, it is evident from the results represented in table 1 which are more efficient in terms of the gain in PSNR, and of visual quality, compared to the methods already interpreted "VisuShrink", that can be explained by the concept of adaptation of the threshold used for each level and each decomposition subband.

This can be interpreted by the use of the optimal thresholds obtained for each subband as well as the thresholding (activation) function used.
Out of all the test images, the best improvement in peak signal-to-noise ratio was obtained by the method proposed in section 2 compared to the methods described above.

It is quite clear, that the method proposed in the 2nd section is the most efficient for all the test images, from the point of view of noise reduction, high values of the PSNR, and also the non generation of artefacts on the reconstructed images (see the images of the figures (see figure [06 - 07])). Indeed, the study was able to show a relative superiority of the proposed method.

In addition, we note a finding that can be drawn from the experimental results obtained that there are several factors affecting the performance of the denoising methods discussed previously, namely the dimension, the content of the image and the variance of the noise. . However, these factors do not influence the performance of the proposed method.

In other words, the proposed algorithm always performs well regardless of the size of the image, its content and the variance of the noise contained in the image. But despite the difference between these two methods and the BayerShrink being much better, the Random Forest method shows good results that are close to the latter and sometimes even exceed them.

\section{Conclusion}

The work presented in this research aimed at the denoising of images by artificial intelligence techniques, where two methods have been implemented among which one based on decision trees has been proposed. Is given too a general overview on the Wavelet Transform as well as on the decision trees (Random Forest algorithm).

The extension of denoising, as proposed by DONOHO, based on the thresholding of wavelet coefficients, represents a reference for denoising techniques with thresholding .

In order to study the performance of the various methods selected, several experiments were carried out. The comparative study established is based on the calculation of the peak signal to noise ratio (PSNR) and the mean square error (MSE) as well as on the visual quality of the denoised images.

Through the results obtained, we have clearly seen the complete superiority of techniques based on artificial intelligence (Random Forest) for the reduction of additive noise, operating on all the test images used.

The comparative study showed the superiority of the proposed method, where we used the decision trees to obtain the optimal thresholds and the application of the Random Forest algorithm to improve the quality of the denoised image. For this, we can conclude that we have successfully used decision trees for the optimization of thresholds for each subband in the denoising domain of satellite images.

The perspectives and directions of research to be followed in order to improve our results relate both to the calculation of the threshold and to the wavelet decomposition. In particular, we believe: kind.

Investigate new methods of estimating noise of any

Estimate the noise in the frequency domain through a representation by local maxima. 
I Investigate other wavelet representations, particularly complex ones.

Future research of satellite image denoising can be done using more complex algorithms, such as neural networks [27] and Rl-Gl-Caputo Method [28].

\section{Acknowledgement}

This work was experimented principally as part of à master project's within University of Oran 1 with the students Bahri Madjid and Moussaab Amrouche [24].

\section{References}

[1] A. Buades, B. Coll and J. M. Morel, "Image Denoising Methods A New Nonlocal Principle" Society for Industrial and Applied Mathematics 2011.

[2] A. Graps, «An introduction to Wavelets,» IEEE Computational Science and, vol. 2, $\mathrm{n}^{\circ} \% 12,1995$.

[3] J. BIGOT, Analyse par ondelettes, Université Paul Sabatier Toulouse III, 2009.

[4] Jean-Michel Morel, «Image denoising and the structure of images,» chez Séminaire de Stéphane Mallat, france, 2018.

[5] S. A. P. T. Mir, «Satellite Image Denoising Using Discrete Cosine Transform.,» Indonesian Journal of Electrical Engineering and Informatics (IJEEI), vol. 4, n% \% 15, pp. 372-375, 2017.

[6] L. Breiman, «Random forests,» Machine Learning, vol. 45, pp. 5-32, 2001.

[7] N. Sirikulviriya et S. Sinthupinyo, «Integration of rules from a random forest,» chez International Conference on Information and Electronics Engineering IPCSIT, Singapore, 2011.

[8] R. Vani et S. Rajan K, «Effective satellite image enhancement based on the discrete wavelet transform.,» International Journal of Business Information Systems, vol. 4, n \%133, pp. 446-471, 2020.

[9] Singh SK, Singh KB, Singh VI. Medical Image De noising In The Wavelet Domain Using Haar And Db3 Filtering. International Refereed Journal of Engineering and Science (IRJES). September 2012; 1(1):01-8.

[10] Jumah A. Denoising of an Image Using Discrete Stationary Wavelet Transform and Various Thresholding Techniques. Journal of Signal and Information Processing. Feb 2013; 4(1):33-41.

[11] Om H, Biswas M. An Improved Image Denoising Method Based on Wavelet Thresholding. Journal of Signal and information Processing. Sept 2012; 3(1):109-16.

[12] Kaur G, Kaur R. Image De-Noising using Wavelet Transform and various Filters. International Journal of Research in Computer Science. March 2012;2(2):15-21

[13] Ruikar SD, Doye DD. Wavelet Based Image Denoising Technique. International Journal of Advanced Computer Science and Applications. March 2011; 2(3):49-53.

[14] Sudha S, Suresh GR, Sukanesh R. Speckle Noise Reduction in Ultrasound Images by Wavelet Thresholding based on Weighted
Variancell. International Journal of Computer Theory and Engineering. April 2009;1(1):7-12.

[15] Achim A, Bezerianos A, Tsakalides P. Wavelet-based ultrasound image denoising using an alpha-stable prior probability model , Proceedings of 8th IEEE International Conference on Image Processing Greece: 2001. 2.p. 221-4.

[16] Kaur L, Gupta S, Chauhan R C, Image Denoising using Wavelet Thresholding. 3 Conference on Computer Vision, Graphics and Image Processing. India:2002. p. 16-8.

[17] Mohideen SK, Perumal SA, Sathik MM, Image De-noising using Discrete Wavelet transform. International Journal of Computer Science and Network Security (IJCSNS). January 2008;8(1):213-6.

[18] Ramani S, Blu T, Unser M, Monte-Carlo SURE: A blackbox optimization of regularization parameters for general denoising algorithms, IEEE Transactions on Image Processing. Sept 2008;17(9):1540-54.

[19] Wilson, Roland, Rajpoot, Nasir M. Image volume denoising Using a Fourier-wavelet basis. 6th Baiona Workshop On Signal Processing In Communications , Baiona, Spain, Sep 2003.

[20] Rakheja P, Vig R. Image Denoising using Combination of Median Filtering and Wavelet Transform. International Journal of Computer Applications. May 2016; 141(9):31-5.

[21] Mohideen K , Perumal A, Krishnan, Sathik M .Image Denoising And Enhancement Using Multiwavelet With Hard Threshold In Digital Mammographic Images. International Arab Journal of eTechnology.Jan 2011; 2(1):49-55.

[22] Chang S, Grace, Yu B, Vetterli M. Adaptive wavelet thresholding for image denoising and compression. IEEE transactions on image processing. Sept 2000; 9 (9): 1532-46.

[23] Veena P V, Devi G R, Sowmya V, Soman K P,Least Square based Image Denoising using Wavelet Filters. Indian Journal of Science and Technology. Aug 2016; 9(30):1-6.

[24] Bahri Madjid, Moussaab Amrouche, Débruitage de fréquence spatial avec seuil de Distribution gaussienne adaptative généralisée Amélioré dans le domaine des ondelettes d'image Satellitaire, PFE of Master, University of Oran 2, Algeria, 2021.

[25] Etat de l'art sur les techniques de débruitage dans le traitement d'image, Sarah Benziane, séminaire international sur les mathématiques et l'informatique 5 (2), 335-339

[26] Soltani, O. (2011). Restauration d'Images satellites via la transformée en ondelette (Doctoral dissertation, Université de Batna 2).

[27] Zhang, F., Cai, N., Wu, J., Cen, G., Wang, H., \& Chen, X. (2018). Image denoising method based on a deep convolution neural network. IET Image Processing, 12(4), 485-493.

[28] Ganga, M., Janakiraman, N., Sivaraman, A. K., Vincent, R., Muralidhar, A., \& Ravindran, P. (2021). An Effective Denoising and Enhancement Strategy for Medical Image Using Rl-Gl-Caputo Method (Vol. 38, pp. 402-408). Advances in Parallel Computing (Smart Intelligent Computing and Communication Technology), IOS Press.

\section{Creative Commons Attribution License 4.0 (Attribution 4.0 International, CC BY 4.0)}

This article is published under the terms of the Creative Commons Attribution License 4.0 https://creativecommons.org/licenses/by/4.0/deed.en US 